\title{
Vaccine or mask policy: one Epidemiologists opinion on the ethics of this healthcare policy
}

\section{Editorial}

Recently, several healthcare organizations in Maine have initiated an influenza policy where healthcare workers can either get the seasonal vaccination or wear a mask for the season. This has been challenged by some of the healthcare staff as an invasion of personal liberties. Few can argue against the positive effects associated with decreasing suffering and improving life span as a result of public health initiatives. Arguably, the most successful of these initiatives is prophylaxis against infectious disease, including hand washing and vaccinations. In order to enjoy optimal health, both as individuals and as a society, people must receive the optimal health care services, and these must be coordinated effectively, based in population health. According to many researchers, avoiding patient harm has become a major health concern, and remains a leading cause of death in the United States. ${ }^{1}$ Moreover, the need to vaccinate health workers has been reasonable well established for over a decade, as outlined by Poland. ${ }^{2}$ There is also precedence in the concepts of standard of care, whereby patients being treated should reasonably expect those providing the treatment to have taken practical steps to avoid causing further harm. As Field ${ }^{3}$ points out, from a public health and patient centered standpoint, vaccination is a very minor procedure (where the person is not medically prohibited from being vaccinated). This minor procedure greatly reduces the risk of infecting patients, fellow health workers, and persons who are medically prevented from receiving the vaccine (either an allergy to egg, or any ingredients in the vaccine, or a history of Guillain-Barre Syndrome).

During the flu season 2015-2016, over 5.1million illnesses, 2.5million medical visits, 71,000 hospitalizations, and over 3,000 deaths were attributed to influenza. ${ }^{4}$ These were largely preventable, and the hospitalizations, and deaths were for some avoidable. Requiring an annual influenza vaccine for health workers protects both the staff and our patients, many of who may already suffer compromised health or weakened immune response. Heard immunity, ${ }^{5}$ the percentage of vaccinated members of the community that help protect those who are not (or cannot be) vaccinated depends on high vaccination rates, and maintaining health. Health care workers are at a far greater risk of contracting and spreading infectious disease. According to Galanakis, ${ }^{6}$ the gulf between the desired levels of vaccination coverage is so low for voluntary vaccination, as to make mandatory vaccination both reasonable and necessary. While some may feel that vaccination is a personal liberty decision, and that a mask may suffice in place of immunization, the question must be asked if the healthcare worker indeed has the best interest of the patient in mind. ${ }^{7}$ With this understanding, and the desire to maintain benevolence, the argument against influenza vaccination for healthcare workers not medically prevented from such vaccinations is both illogical and dangerous. As a Clinical Epidemiologist, I fully support the use of annual vaccination for influenza (and other age related vaccines). Finally, as suggested by Bloom, ${ }^{8}$ requiring healthcare workers is not government overreach, but given the availability of opportunistic infections in hospitals and

\author{
Volume 6 Issue 6 - 2017

\section{Sumner Davis W} \\ Community Psychologist and Clinical Epidemiologist, Davis \\ Health Consulting, USA
}

Correspondence: W Sumner Davis, PhD, MPH, MSHS, USA, Tel 2074654698,Email drwdavis@me.com

Received: December 04, 2017 | Published: December I2, 2017

clinics, the financial impact of treating influenza cases, and the number of deaths occurring from influenza each year, mandatory vaccination for healthcare workers is not just wise, it's common sense. The use of masks is at best a feel-good response that may not significantly decrease the spread of influenza from patient to patient unless other infection protocols are rigidly enforced.

\section{Acknowledgements}

None.

\section{Conflict of interest}

Author declares that there is no conflict of interest.

\section{References}

1. Card AJ. Patient safety: this is public health. $J$ Healthc Risk Manag. 2014;34(1):6-12.

2. Poland GA, Tosh P, Jacobson RM. Requiring influenza vaccination for health care workers: Seven truths we must accept. Vaccine. 2005;23(17-18):2251-2255.

3. Field RI. Mandatory vaccination of health care workers: whose rights should come first? PT. 2009;34(11):615-618.

4. Rolfes MA, Foppa IM, Garg S, et al. Estimated Influenza Illnesses, Medical Visits, Hospitalizations, and Deaths Averted by Vaccination in the United States. Centers for Disease Control and Prevention. USA; 2016.

5. Fine P, Eames K, Heymann DL. Herd immunity: a rough guide. Clin Infect Dis. 2011;52(7):911-916.

6. Galanakis E, Jansen A, Lopalco PL, et al. Ethics of mandatory vaccination for healthcare workers. Euro Surveill. 2013;18(45):20627.

7. Teitelbaum J, Pozgar G. Law, ethics, and policy in healthcare administration. (Custom edn) USA: Jones \& Bartlett Learning; 2015.

8. Bloom J. Mandate the flu vaccine for healthcare workers. US News and World Report, USA; 2013. 